\title{
Outcome of patients with initial non-mechanical ventilation management in severe pneumonia
}

\author{
A Ortín*, R Jiménez, S Rebollo, L Herrera, S Moreno, A Fernández, M Galindo, J Murcia, L Tárraga, A Ojados, \\ OM Téllez, M Contreras, MD Rodríguez, JM Allegue \\ From ESICM LIVES 2015 \\ Berlin, Germany. 3-7 October 2015
}

\section{Introduction}

Stablishing when a non-invasive first line approach can be an option in the management of acute respirarory failure due to severe pneumonia represents a major challenge.

\section{Objectives}

To analyze the influence of first line non-invasive respiratory support in the outcome of patients admitted with severe pneumonia.

\section{Methods}

We analyzed retrospective data from patients admitted due to severe pneumonia in our polivalent ICU during a 4-year period. Every patient received antibiotic treatment according to current guidelines.

We collected data related to the specific initial ventilatory therapy, need for therapy escalation and clinical outcomes.

\section{Results}

During the period studied, 113 patients were admitted to our ICU due to severe pneumonia (78 \% from community and $22 \%$ from hospital) $36(31.8 \%)$ were treated initially with invasive ventilation (MV), 45 (39.2\%) with non-invasive ventilation (NIV), 5 (4.4\%) with highflow nasal cannula (HFNC) and 27 (23.9\%) with conventional oxygen therapy (COT).

NIV was applied to older and more sick (as measured by APACHE II) patients compared to HFNC and COT [63.3 years (CI 95\% 58.2-68.5) vs 48.3 (41.5-55) p 0.001; APACHE 17.8 (16.1-19.4) vs 14.9 (12.8-17) p 0.03] ICU stay and mortality was higher in NIV group compared

Hospital General Universitario Santa Lucía, Servicio de Medicina Intensiva, Cartagena, Spain

(c) 2015 Ortín et al.; This is an Open Access article distributed under the terms of the Creative Commons Attribution License (http:// creativecommons.org/licenses/by/4.0), which permits unrestricted use, distribution, and reproduction in any medium, provided the original work is properly cited. to conventional therapy [8 days (IQR $5-18)$ vs 4 (2-11) p 0.004; mortality $35.5 \%$ vs $9.1 \%$ p 0.007 ]

Higher rate of delayed intubation was observed in NIV patients, not reaching statistical difference between groups ( $40 \%$ vs $24.2 \%$ p 0.15) In those with delayed intubation, duration of MV was higher [13 days (IQR 626) vs 8 (3-11) p 0.006] Those patients requiring escalation to mechanical ventilation presented, as was expected, double mortality (50\% vs $11.5 \%$ p 0.001 )

Among all patients finally ventilated (63 patients), those with delayed MV had similar age an APACHE II but longer ICU and hospital stay and duration of mechanical ventilation [13 (5-17) vs 19 days (13-39) p $0.005 ; 18$ (12-25) vs 30 days (15.5-47.5) p 0.032; 8 (3-11) vs 13 days $(6-26) \mathrm{p} 0.006$ ] and a trend to higher mortality (46.4 vs $34.3 \%$; ns).

\section{Conclusions}

An initial non-invasive oxygen therapy management for severe pneumonia did not impact in outcomes, except for patients finally requiring mechanical ventilation. Non invasive ventilation was probably applied to sicker patients and this fact may explain the worse evolution of this subgroup.

Published: 1 October 2015

\section{References}

1. Antonelli M: Crit Care Med 2007, 35:18-25.

2. Murad A: J Crit Care 2015, 30(1):49-54, Feb.

Sztrymf B: J Crit Care 2012, 27(3):324.e9-13, Jun.

4. Antonello N: J Crit Care 2015, Mar 5.

doi:10.1186/2197-425X-3-S1-A95

Cite this article as: Ortín et al:: Outcome of patients with initial nonmechanical ventilation management in severe pneumonia. Intensive Care Medicine Experimental 2015 3(Suppl 1):A95. 